\title{
Rearch on Remote Video Monitoring Technology in Industry based on Zigbee
}

\author{
Li Wei Wang \\ Teacher's College, Beihua University, Jilin City, China
}

\begin{abstract}
ZigBee is a recent rise of wireless network technology, with low-rate, low cost, low power, network capacity and other characteristics, to organize their own network, and network recovery ability, very suitable for wireless sensor networks. In this paper, the industrial production environment, systematic study of the ZigBee technology, video compression technology, as well as data communication principle, the development of the industrial video monitoring and control system based on ZigBee protocol.
\end{abstract}

\section{General instruction}

With the development, technological advances, modern industrial production scale expanding rapidly update production equipment, production technology becomes more advanced, accompanying the increasingly complex production environment, production monitoring and control technology which made more high requirements. Traditional wired network monitoring and control system by wiring, maintenance and scalability limitations, cannot better adapt to modern industrial production environments, requiring more advanced industrial monitoring and control system to meet the needs of modern industrial production. Wireless sensor network is a major trend of modern communication development, has a certain application basis, and in the continuing efforts of researchers, more advanced and mature, is an alternative to a wired network the best choice.

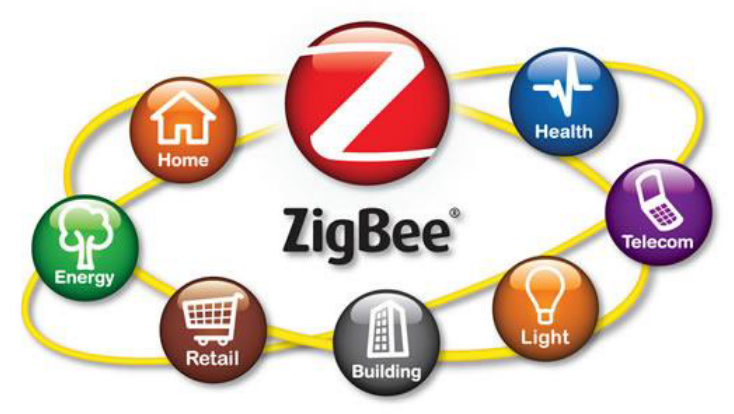

Figure 1. ZigBee wireless Network Protocol chart

\section{Wireless communications in early industrialization}

The first generation of wireless communications in early industrialization, from war needs, people use smoke signals, fire, semaphore, gestures to fellow transmit information. Of course, the amount of information and complexity is low, and information transmission is within visible distance range. In order to achieve a greater amount of information transmission, richer, more distant, it is encoded in the form of information, the formation of the original signal. Set up observatories in line of sight and at receiving the message transmitted to the distance again, that the relay-style messaging. Until 1838, the United States - Samuel Morse (Samuel Finley Breese Morse) invented the Morse code (also known as the Morse code), electricity yard pass digital message, the digital and the word corresponding to realize the complex information pass, and is the earliest form of digital communication, the end of the original forms of information transmission. Subsequently, the Americans Bell invented the telephone in 1876, replacing the telegraph. It took another ten years, in 1895, Italy's Galileo Stockholm • Marconi in his father's estate began his experiment, successfully received a radio signal from the 1.5 miles (about $2.4 \mathrm{~km}$ ) outside realization the world's first wireless telegraph transmission, thus, the birth of modern wireless communications.

As of 1934, the United States nearly 200 city police monitoring system, fifty-eight state police used AM (Amplitude Modulation, AM) technology for wireless communication system so that public safety be better protected. According to statistics, the mid thirties of the last century, there are about more than five thousand mobile devices installed radio receiver, based mainly in the vehicle, for the purposes of these early radio device mounted on a vehicle, the noise of the vehicle is a major obstacle. Later, an American radio engineer EdwinHoward Armstrong (Edwin Howard Armstrong) invented the frequency modulation (Frequency Modulation, FM) 
technology; its powerful features make it the world's communications major modulation techniques.

World War II accelerated the development of manufacturing and micro technology, and has been used in a large number of one-way and two-way radio communication systems and television systems. With the advancement of technology, the development, wireless communication technology has been extended too many fields such as business, agriculture, industry, etc., and the depth and breadth of applications evolving.

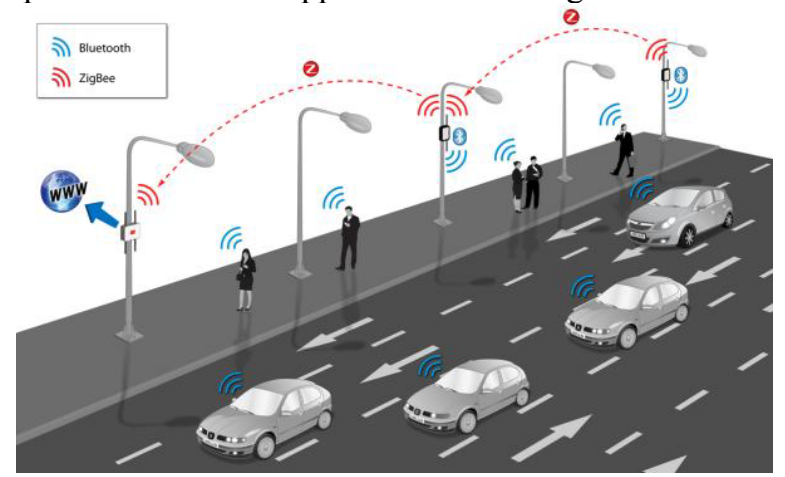

Figure 2. zigbee and Bluetooth match results demonstrate

\section{State of the art industrial monitoring and control}

Currently widely used industrial monitoring and control technology is a network monitor; it evolved from earlier centralized monitoring technology came. Centralized monitoring technology mainly through the central control platform for industrial production of core equipment for centralized monitoring; monitoring equipment is used in large-scale instrumentation. With the expansion of production scale, in the 1950 s, sixties, used in industrial production, a special control room for centralized control of production parameters, such as current signal $(0 \sim$ $10 \mathrm{~mA}, 4 \sim 20 \mathrm{~mA}$ of DC current signal) and voltage signals (1 5VDC voltage signal), and other analogy signals are processed in a centralized control room. The 1960s and 1970s, due to the development of computer technology, cost and operational monitoring equipment limitations, encourage people to try to use a computer to replace the traditional test instrumentation and control terminal, so the digital monitoring and control system began to develop. In the initial stage, the stability, the computer system is limited to the use of computer system architecture to achieve centralized monitoring and control of the production process has not been accepted. A failure occurs because the computer will cause the entire monitoring system failures, causing serious losses to the production, which is very dangerous.

In recent years, with the rapid development of information technology and network technology, Ethernet has become one of the most widely used network technology information, which has been applied to a large area of industrial monitoring and control, Industrial Ethernet is based on the IEEE 802.3 powerful regional and cell networks using TCP / IP communications protocol. By designing real-time communication methods and high stability industrial application network, Ethernet can meet the needs of real-time industrial data communications, industrial control functions to achieve the top-level data and the underlying control integrated together. Industrial Ethernet relative to the early industrial automation technology, transmission speed, construction costs, ease of formation and compatibility and so there are a lot of advantages. But established on which the monitoring and control system for real-time communication, network stability, system security, and data integrity have high demands, because monitoring technology Industrial Ethernet is for industrial production processes.

Wireless communication technology is following the industrial Ethernet and fieldbus, used in industrial production monitoring and control of another hot technology is the development trend of industrial automation production. Wireless communication technology to avoid the traditional wired network defects, wired network needs a conductor media for data transmission, resulting in a series of work and transmission pathways, such as planning the wiring, the default port, line testing, line expansion and so on. With the accompanying destruction of buildings, waste interfaces, maintenance difficulties, difficulties in expansion and other ills. In addition, in some special production environment (such as the petrochemical industry as well as hydrological monitoring, environmental monitoring, oil, coal, street surveillance, meter monitoring, etc.), a wired network coverage and network support is limited, there is high cost. The wireless communications transmission medium is not required in the industrial production environment; you can send data to the space, by installing a network access device in the networking area, wireless Internet connection, high flexibility, scalability, and convenient operation advantage.

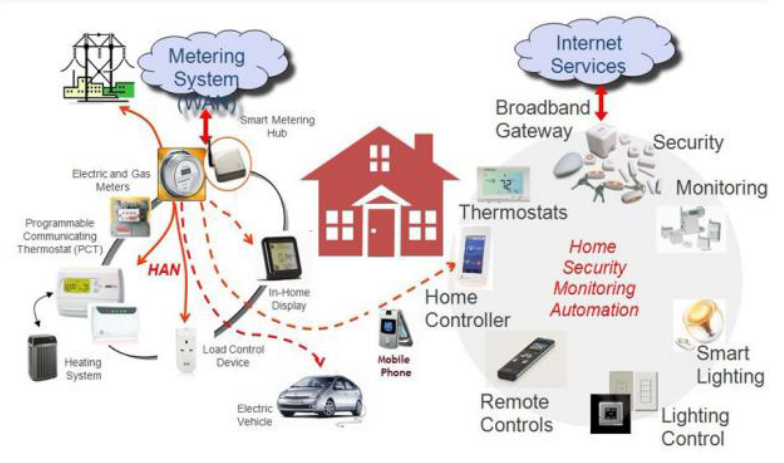

Figure 3. Zigbee remote monitoring system Demo

\section{ZigBee and image compression coding technology}

ZigBee is a wireless network in recent years, the rise of communications technology, the technology alliance by British Invensys, Japan's Mitsubishi Electric, Motorola USA, the Netherlands, Philips Semiconductors and other companies in the development of a network, security and application software protocol layer, cost, power 
consumption, speed, complexity is relatively low, when the word is used to notify the pollen from the honey bees companion way location, is a simple, effective "Wireless Communications" in the form of.

ZigBee technology is mainly used in short-distance data transmission between a variety of electronic devices, the lower its data transfer rate, and therefore suitable for industrial production environments wireless transmission of video data. Its main features are as follows has several areas:

(1) The rate is low. ZigBee network in all the different bands work data transfer rates are at a low level, but also for different working frequencies corresponding rate is adjusted, so that work more in line with the principle of energy distribution.

(2)Low power Because the transmission rate is low, the transmission power is only $1 \mathrm{~mW}$, and the use of sleep mode (when required data is received, only wake up by a network of "coordination" on them), so the ZigBee technology is very low power consumption, for energy security network to run a small, usually two AA batteries can support up to two years of operation of the network.

(3)Low cost Compared to other short-range communication technology, ZigBee technology research and development costs low, because of its simple design protocol stack. Moreover, program development is also very convenient; eight microprocessors will be able to meet the needs of ordinary network. With the application and development of the product, is expected to further reduce costs.

(4) Reliable data transmission. Using CSMA-CA (Carrier Sense Multiple Access-Collision Avoidance) mechanism to avoid a collision, and dedicated time to leave gaps for the fixed-band communications to provide protection, but also the establishment of independent network, data communication between nodes in the network have robust path transmission security, because the transmission path is not fixed, the flexibility to choose the best communication path through a router.

(5)Short delay for latency applications optimized data communication with a relatively short delay and a short wake-up delay.

(6)The network capacity ZigBee network can be employed, such as a star, the number of state and mesh network structure, can form large networks with a hierarchical network structure.

(7)Network security ZigBee technology uses a common encryption algorithms, and provides a complete data packet check function (available on the quality of data packet inspection, such as redundancy and integrity), thus ZigBee network has very good security.

\section{Requirements ZigBee network transmissions of video compressed data}

ZigBee is a low-rate wireless data transmission network, and the content of the original uncompressed video data is the large number of successive image frames, which have higher bandwidth data transmission requirements, so the transmission of video in low transmission rate of ZigBee networks the data must be through efficient compression, otherwise, is not complete real-time video monitoring tasks. Video compression is by deleting unnecessary video data (such as duplicate colors, color changes, repeating image frames, etc.), so as to achieve the purpose of reducing the amount of data. Video compression is divided into loss and lossless compression, loss compression of video information deleted when decompression is unrecoverable, but it can greatly reduce the amount of video data, and lossless compression during decompression can restore the original video data, the picture quality can be good assurance, but its share of the memory space will not be reduced. This article video application environment for production and management industry, the core purpose is to monitor and control, so the picture quality requirements can be appropriately lowered, no need to reach the TV, movies, etc. to quality requirements, so the use of loss compression is most appropriate.

Compressed video data is a measure of the effectiveness and versatility compression method selection criteria. Moment, major international organizations to develop video coding standard is the International Telecommunication Union Telecommunication Standardization Organization (ITU-T for ITU Telecommunication Standardization Sector) and the International Organization for Standardization/ International Electro technical Commission (ISO/IEC, International Organization for Standardization/ International Electro technical Commission) , MPEG-x and H.26x series of video compression standard is developed by the two, two series of compression encoding technology is relatively similar, including MPEG-4 and H.264 compression technology is the most studied and most widely used. MPEG-4 may be employed according to the different objects of different coding algorithm, coding algorithm is more flexible, more targeted, thus making the compression rate, encoding target independent and allows reuse of video data, in addition, MPEG-4 encoding in different objects The rate can be flexibly assigned, which can be assigned to match the byte coded according to the degree of importance, the important objects of points on the object of little minor points, so you can achieve good results at low bit rates. The H.264 encoding technology used is more advanced than the MPEG-4, but it's relatively complex computing, technology is not mature enough, the risk of instability exists in the coding system. In summary, to meet ZigBee network video transmission, the paper selected coding technology MPEG-4 format, industrial surveillance video compression.

\section{Image compression coding algorithm}

MPEG-2 technology has been developed MPEG-4, MPEG-4 compression algorithm uses a large number of 
efficient algorithms, but also provides specialized tools for the operation and the coded video data, so the MPEG4 video standard than the earlier of a higher compression ratio, better scalability advantages, MPEG-4 not only uses such as motion estimation and motion compensation and some other traditional methods, but also uses a video extraction, classification and other advanced video encoding technology, based on manipulation of the image frame is not again limited. In addition, flexible rate control video object is achieved in accordance with the importance of the distribution rate, even if the bandwidth is not ideal, but also to ensure picture quality, picture frames no longer limited by the low bit rate. Therefore, the full bandwidth utilization is better quality.

MPEG-4 video standard introduces the video object (Video Object, VO) concept can be based on content access, high efficient video data compression. A video sequence is split into one or a group of $\mathrm{VO}, \mathrm{VO}$ can be any shape in the video area (such as Portrait, things like), the user can be obtained and image manipulation entity, it is flexible and no longer limited to the traditional rectangular frame, VO has a moving object, the object shape and texture objects 3 types. Furthermore, MPEG-4 also introduces a video object plane (Video Object Plan, VOP) concept, VOP is a VO successive frames having image frame, such as $\mathrm{VO}$ is defined as a ball beating, then the VOP is the ball in the beating during a time of the image. VOP is the basis for MPEG-4 encoding, encoding or interpolation can be independently encoded, i.e. estimated or motion picture compensation did not occur. VOP before the MPEG-4 coding needs to be defined.

\section{References}

1. Li Xinrong overall intelligent building control system design $[\mathrm{J}]$ Technology Information, 63(2007)

2. Jiang Xiaolin, Miao $\mathrm{Yu}$ design and development of intelligent building monitoring system. Security Technology, 23(2006)

3. Wang Shuang application and development of video surveillance systems in industrial production. Network and Information, 25(2009)

4. Sun school on behalf of the development and application of "video surveillance system. Jiangxi Communication Technology, 38(2009)

5. Ruan Weihua common indoor wireless location technology introduction. Science and Technology Information, 90(2009)

6. Liang Guangsheng, Liudan Juan, Haofu Zhen based on CC2430 ZigBee wireless network nodes. Electronic Design Engineering, 18(2010)

7. Mengzhen Fei, Ya Ling, Hou Yi handsome design is based on ZigBee technology wireless module. Electronic Component Applications, 39(2010)

8. Baozhao Wei, Wang Lin, Xu Qideng design USBRS232 converter module FT232R. Computer Knowledge and Technology, 1204(2008)
9. Wang LonWorks technology based on LabVIEW and iLab remote experiment system design. Dalian: Dalian University of Technology 251(2009) 